\title{
Normalization using ploidy and genomic DNA copy number allows absolute quantification of transcripts, proteins and metabolites in cells
}

\author{
Hiroshi Shimada ${ }^{*}$, Takeshi Obayashi², Naoki Takahashi ${ }^{3}$, Minami Matsui ${ }^{3}$, Atsushi Sakamoto ${ }^{1}$
}

\begin{abstract}
Background: Quantification of transcripts, proteins, or metabolites is straightforward when the factor used to normalize these values remains constant between samples. However, normalization factors often vary among samples and thus must be developed for each new analytical method.

Results: We demonstrate quantification of transcript and protein levels in Arabidopsis based on genomic DNA copy number. We extracted total nucleic acid from 3-week-old rosette leaves of wild-type Arabidopsis and the pale-green/dwarf mutant, $a b c 4$, and quantified the number of transcripts by quantitative reverse-transcription PCR using genomic DNA copy number and ploidy (as determined by cytometry) for normalization. Our data indicated that normalization using genes commonly employed as references resulted in inaccuracies in transcript levels of the genes RBC-L and RBC-S (encoding the large and small subunits, respectively, of ribulose 1,5-bisphosphate carboxylase/oxygenase) in wild type and mutant. Normalization using genomic DNA copy number and ploidy, however, appropriately showed that the RBC-L and RBC-S transcript levels per cell in the mutant were significantly lower than that in wild type. Furthermore, quantification revealed that a cell of a 3-week-old wild-type Arabidopsis rosette leaf had an average of $7.5 \times 10^{3}$ transcripts of RBC-L, $9.9 \times 10^{3}$ transcripts of RBC-S, and $1.4 \times 10^{6} 18 S$ rRNA. We similarly analyzed the accumulation of RBC-L and LHCP (light-harvesting chlorophyll $a / b$ protein) in wild type and mutant based on ploidy and genomic DNA copy number that was determined by direct quantitative PCR analysis of extracts using a DNA polymerase tolerant to a wide range of common PCR inhibitors. Furthermore, we estimated the number of RBC-L molecules $\left(2.63 \times 10^{8}\right)$ and chlorophyll molecules $\left(1.85 \times 10^{9}\right)$ in each cell in 3 -week-old wild-type rosette leaves; these values had relatively low coefficients of variation, underscoring the reliability of our method.
\end{abstract}

Conclusion: Genomic DNA copy number and ploidy are useful as general normalization factors, providing an easy method for determining the number of transcripts, proteins, and metabolites in a cell.

\section{Background}

Cellular levels of transcripts, proteins, and metabolites are usually quantified relative to the value for a known, constitutively expressed cellular factor. Quantification of transcripts using northern hybridization is based on total amounts of RNA or mRNA. Quantification of transcripts using RT-PCR analysis, including real-time RT-PCR, is based on the expression level of a reference gene [1-4], and a DNA array detects

\footnotetext{
* Correspondence: hshimada@hiroshima-u.ac.jp

'Department of Mathematical and Life Sciences, Graduate School of Science, Hiroshima University, 1-3-1, Kagamiyama, Higashi-Hiroshima, 739-8526, Japan Full list of author information is available at the end of the article
}

relative levels of transcripts [5,6]. Protein levels are typically quantified by Coomassie Brilliant Blue (CBB) staining of samples subjected to SDS-PAGE, by two-dimensional difference gel electrophoresis for proteome analysis, by immunoblotting, or by enzymelinked immunosorbent assay relative to the weight of total protein, fresh weight, dry weight or culture volume. Metabolites are often quantified based on the weight of total protein, fresh weight or dry weight. Such quantification methods are useful when the normalization factor does not vary among samples. Between tissues, however, the transcriptional activity may differ, and the ratio between mRNA and rRNA

\section{C) Biomed Central}


may vary widely depending on the cell population $[7,8]$. Because rRNA comprises a large proportion of total RNA in the cell, transcript quantification based on total amounts of RNA or mRNA in one cell type may not accurately reflect the transcript levels in other cell types. The precision of quantitative (q)RT-PCR depends on accurate transcript normalization using constitutively expressed genes. Statistical algorithms have been developed to help validate reference genes $[3,4]$; prior to analysis, however, it is difficult to know which reference gene is consistently expressed among the samples, such as when a novel mutant or treatment analysis is under consideration. Similarly, total protein, fresh weight, dry weight, or culture volume may vary between samples.

In Arabidopsis thaliana, abc4 is a mutant of the phylloquinone biosynthesis gene and exhibits the dwarf and pale-green phenotype [9]. The mutant has fewer chloroplasts than wild type, and the intercellular space is also larger [9]. Northern hybridization using total RNA revealed that the $R B C-L$ (Rubisco large subunit) and $R B C-S$ (Rubisco small subunit) transcript levels are significantly elevated in the $a b c 4$ mutant, whereas the LHCP (light-harvesting chlorophyll $a / b$ protein) transcript level is almost the same as in the wild type [9]. Quantification by CBB staining of samples subjected to SDS-PAGE or by immunoblotting based on total input protein revealed similar levels of both RBC-L and RBC-S between wild type and $a b c 4$ and that the mutant had a slightly reduced level of LHCP [9]. To address these potentially confounding factors in quantitative analysis, we developed methods to quantify transcript, protein, and metabolite levels based on genomic DNA copy number and ploidy using A. thaliana wild type and $a b c 4$.

\section{Results and Discussion}

\section{Analysis of genomic DNA copy number per cell}

Many plant cells have a unique cell cycle mode with cells undergoing iterative DNA replication without cytokinesis. This endoreduplication is frequently observed in some, but not all, plants [10]. Flow cytometry demonstrated that $32 \%$ of nuclei isolated from 3 -week-old wild-type rosette leaves were in the $2 \mathrm{C}$ peak, and $68 \%$ of nuclei were in the $4 \mathrm{C}, 8 \mathrm{C}$ and $16 \mathrm{C}$ peaks (Figure $1 \mathrm{~A}$ and $1 C)$. The mutant $a b c 4$ had a dwarf/pale-green phenotype (Figure 1B), as expected [9]. Flow cytometry demonstrated that $50 \%$ of nuclei isolated from 3-weekold $a b c 4$ rosette leaves were in the $2 \mathrm{C}$ peak, and $50 \%$ of nuclei were in the $4 \mathrm{C}$ and $8 \mathrm{C}$ peaks (Figure $1 \mathrm{C}$ ). Therefore, the mean ploidy of 3-week-old rosette leaves from wild type and $a b c 4$ was $4.35 \pm 0.08$ and $3.08 \pm 0.03$ (mean \pm s.d.), respectively.

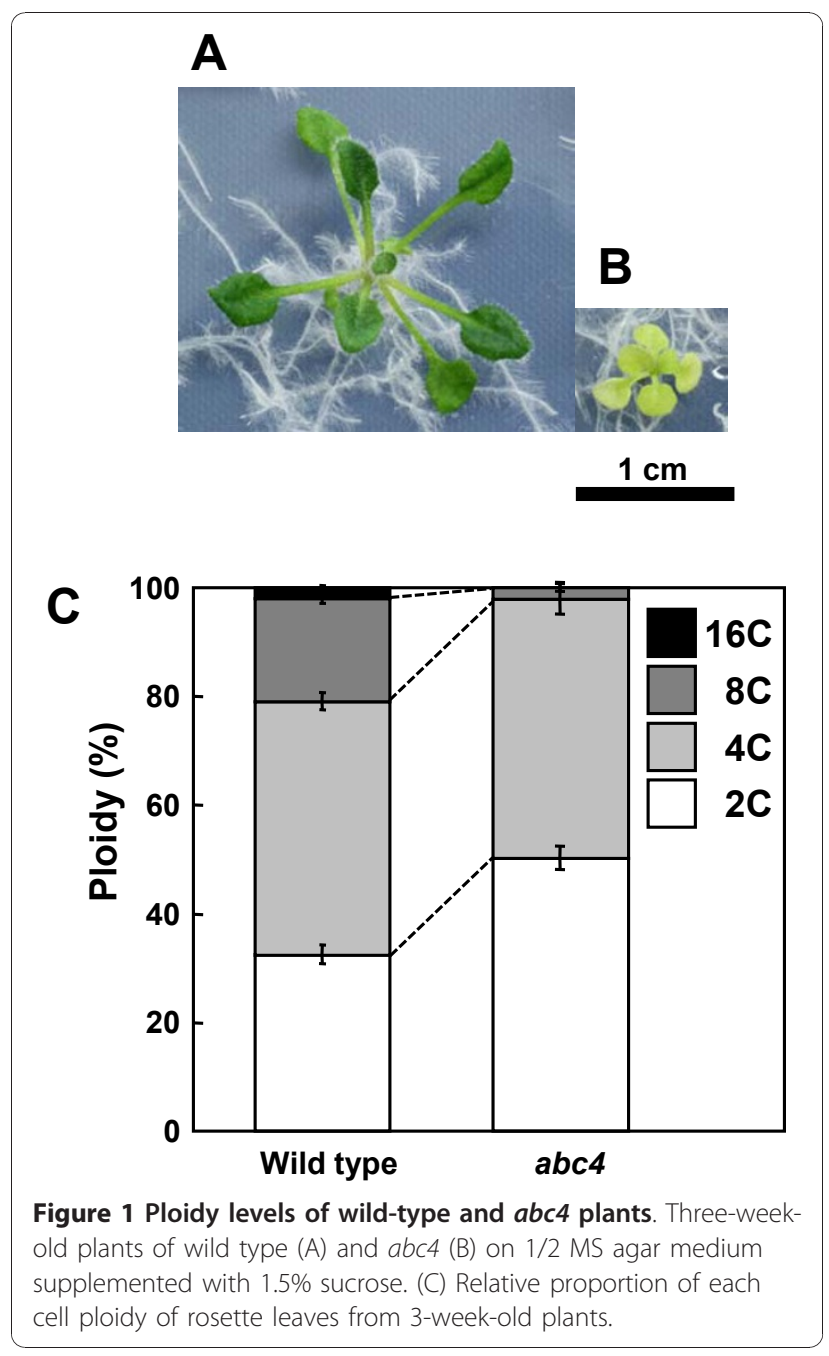

Transcript accumulation normalized to genomic DNA copy number and ploidy

We used qRT-PCR to compare transcript levels of $R B C-L$ and $R B C-S$ between wild-type and $a b c 4$ plants. For quantification using the $\Delta \Delta C$ t method [11], one of several established housekeeping genes, namely ACT2 (actin 2), PDF2 (transposable element gene), $S A N D$ (SAND family protein), GAPDH (glyceraldehyde 3-phosphate dehydrogenase), $U B C$ (ubiquitin-conjugating enzyme), $E F-1 \alpha$ (elongation factor 1- $\alpha$ ), $P P R$ (pentatricopeptide repeatcontaining protein), YLS8 (yellow-leaf-specific protein 8), $U B C 9$ (ubiquitin-conjugation enzyme E2), or the $18 \mathrm{~S}$ rRNA gene or genomic DNA (Figure 2A), was used as the reference (see additional file 1 ). Derivation of the $2^{-\Delta \Delta C t}$ equation, including assumptions, experimental design, and validation tests, is described in the Applied Biosystems User Bulletin \# 2 http://www3.appliedbiosystems.com/ cms/groups/mcb_support/documents/generaldocuments/ 
cms_040980.pdf. When genomic DNA was used as reference, the relative transcript accumulation was calculated as follows: $2^{-\Delta \Delta C t} \times \frac{G_{\text {mutant }}}{G_{\text {wild }}}=2^{-\Delta \Delta C t} \times \frac{3.08}{4.35}$, where $G_{\text {mutant }}$ and $G_{\text {wild }}$ are the genomic DNA copy number per cell (i.e., mean ploidy) of the $a b c 4$ and wild-type plants, respectively (see additional file 2). Using $A C T 2$ as the reference, the $R B C-L$ and $R B C-S$ transcripts were lower in $a b c 4$ plants (Figure 2A; also see additional file 3). By contrast, the levels were comparable between wild type and mutant using SAND or GAPDH as the reference, and the levels were higher in the mutant than in the wild type using $U B C, E F-1 \alpha, P P R, Y L S 8$ or $U B C 9$ as the reference. Use of genomic DNA, $18 S \mathrm{rRNA}$, or $P D F 2$ as a reference revealed slightly lower levels of both $R B C-L$ and $R B C-S$ transcripts in the mutant. Figure $2 \mathrm{~B}$ shows the transcript levels of the genes often used as references in $a b c 4$ relative to the wild type using genomic DNA as the reference. This analysis indicated that the $18 S$ and $P D F 2$ transcript levels were similar between the wild type and mutant. The level of $A C T 2$ transcript was significantly higher in the mutant, whereas levels of $S A N D, G A P D H, U B C, E F-1 \alpha, P P R$, $Y L S 8$ and $U B C 9$ transcripts were lower in the mutant. We concluded that differences between the transcript levels of the reference genes in the wild type and mutant (Figure 2B) resulted in apparent differences in $R B C-L$ and $R B C-S$ transcript levels between the wild type and mutant (Figure 2A). In this assay, the wild type and mutant had comparable levels of $18 \mathrm{~S}$ transcript, but $18 S$ expression is not always consistent between cells $[7,8]$.

Northern hybridization using total RNA revealed that the $R B C-L$ and $R B C-S$ transcript levels were significantly elevated in $a b c 4$ [9]. Note that qRT-PCR normalized to the genes often used as references may provide misleading results (Figure 2A). However, qRT-PCR using genomic DNA copy number and the mean ploidy as the reference can provide more accurate information on the level of transcripts per cell.

\section{Quantification of transcript number per cell}

In 3-week-old wild-type plants, we analyzed the number of transcripts by qRT-PCR using genomic DNA as reference (Figure 2C). We determined the DNA copy number by qRT-PCR and calculated the transcript number per cell as follows:

(transcript number / cell)

$=$ (transcript number / genomic DNA copy number $)$

$\times$ (genomic DNA copy number/cell)

$=2^{-\Delta \mathrm{Ct}} \times 4.35$

$=2^{-\{\mathrm{Ct}(\mathrm{CDNA})-\mathrm{Ct}(\text { genome })\}} \times 4.35$
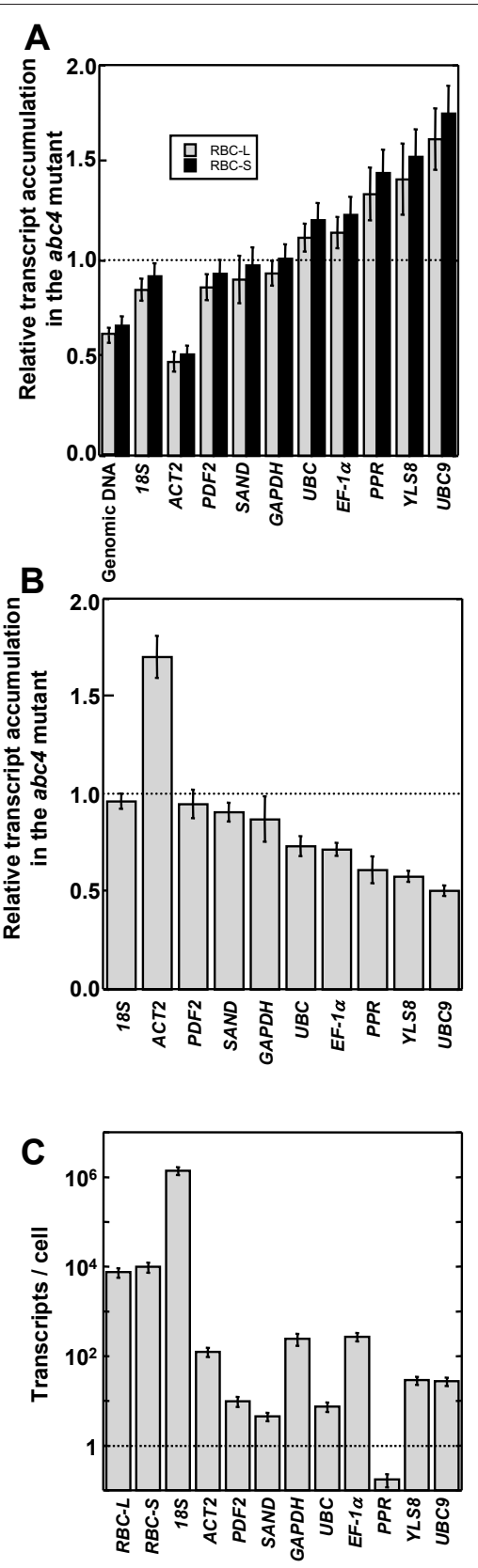

Figure 2 qPCR analysis of $R B C-L$ and $R B C-S$ transcripts in A. thaliana and evaluation of reference genes. (A) Transcript levels of RBC-L and RBC-S in $A$. thaliana rosette leaf samples were analyzed by qRT-PCR and quantified by the $\triangle \triangle C$ t method using the reference genes indicated at bottom. Data reflect relative transcript accumulation in abc4 plants relative to that in wild-type plants. (B) Transcript levels of the reference genes in rosette leaf samples from $A$. thaliana were analyzed by qRT-PCR and quantified by the $\triangle \triangle C t$ method using genomic DNA as the reference. Data reflect relative transcript accumulation in abc4 plants relative to that in wild-type plants. (C) Transcripts numbers per cell in the wild-type A. thaliana rosette leaf were determined relative to genomic DNA using $\mathrm{qPCR}$ analysis. Data reflect the mean \pm s.d. from duplicate experiments of four biological samples. 
Each cell in the wild-type rosette leaf had an average of $7.5 \times 10^{3} R B C-L$ transcripts, $9.9 \times 10^{3} R B C-S$ transcripts, and $1.4 \times 10^{6} 18 S r R N A$ transcripts (see additional file 4). The number of PPR/At1g62930 (pentatricopeptide repeat-containing protein gene) transcripts per cell was much lower (0.17) (Figure 2C; also see additional file 4), indicating that there is less than one PPR transcript per cell. PPR was not transcribed in all cells of wild-type rosette leaves. The rosette leaf contains various cell types (e.g., mesophyll cells, epidermal cells, guard cells and vascular tissue cells), and PPR transcription may be cell type specific. Of course, any gene with less than one transcript per cell should not be used as a reference for quantification of qRT-PCR data.

\section{Protein accumulation normalized to genomic DNA copy number and ploidy}

We analyzed protein expression in 3-week-old wild-type and $a b c 4$ rosette leaves by SDS-PAGE and quantified CBB-stained RBC-L and LHCP bands using ImageJ http://rsbweb.nih.gov/ij/ based on several normalization criteria. When the mass of the total input protein was used for normalization, the RBC-L and LHCP band intensities were nearly identical between wild-type and $a b c 4$ samples (Figure 3A lanes 1 and 2, and Figure 3B), but the amounts of these proteins were slightly lower in $a b c 4$ when fresh weight was used for normalization (Figure $3 \mathrm{~A}$ lanes 3 and 4, and Figure 3B). The fresh weight of all rosette leaves from individual 3-week-old wildtype and abc4 plants was $15.5 \pm 0.3 \mathrm{mg}$ and $3.5 \pm 0.1$ $\mathrm{mg}$, respectively (mean \pm s.e.m., $n=20$ for both). Based on the individual mean fresh weight of rosette leaves, the amount of RBC-L and LHCP protein in abc4 was significantly lower than in wild type (Figure $3 \mathrm{~A}$ lanes 5 and 6, and Figure 3B).

We next quantified protein expression levels normalized to genomic DNA copy number and ploidy established by qPCR using plant extracts. To extract total protein, the plants were homogenized in an extraction buffer containing $10 \mathrm{mM}$ EDTA and 1.0\% SDS, both of which inhibit DNase (see Methods) [12]. Because the KAPA2G Robust HotStart DNA polymerase (Kapa Biosystems) used in this assay is tolerant to a wide range of common PCR inhibitors (e.g., salts and SDS), the protein samples were used without further purification as the qPCR template. Protein samples from equivalent numbers of wild type and mutant cells were then subjected to SDS-PAGE; for this analysis the volume of protein sample from the mutant $\left(V_{\text {mutant }}\right)$ was applied.

$V_{\text {mutant }}=V_{\text {wild }} \times \frac{1}{R} \times \frac{G_{\text {mutant }}}{G_{\text {wild }}}=V_{\text {wild }} \times \frac{1}{R} \times \frac{3.08}{4.35}$, where $V_{\text {wild }}$ is the applied volume of protein sample from wild type, $R$ is the relative genomic DNA copy number of the protein sample from the mutant to that of wild type, and $G_{\text {mutant }}$ and $G_{\text {wild }}$ are the genomic DNA copy number per cell (i.e., mean ploidy) of the $a b c 4$ and wild-type plants, respectively (see Methods and additional file 5). The amounts of RBC-L and LHCP in $a b c 4$ were significantly lower than in the wild type based on genomic DNA copy number (Figure 3A lanes 7 and 8 , and Figure 3B).

\section{Quantification of protein and metabolite number per cell} We next analyzed the number of genomic DNA molecules and RBC-L molecules per cell in protein samples of 3-week-old wild-type rosette leaves. One microliter of a 1:40 dilution of wild-type protein extract and $1 \mu \mathrm{l}$ of plasmid harboring a DNA fragment amplified by PCR (between $2.88 \times 10^{2}$ and $2.88 \times 10^{9}$ molecules, or 0 molecules) were added to the PCR reactions. This mixing procedure was done because the amplification efficiency of the qPCR differed depending on whether the protein extract or the purified plasmid was used as template (efficiency $=0.665$ or 0.890 , respectively). Therefore, we could not extrapolate the genomic DNA copy number in the protein samples using the standard curve created with the purified plasmid. Thus, to estimate genomic DNA copy number, we assessed the effect of exogenously added plasmid DNA in PCR reactions containing genomic DNA on PCR amplification of a DNA segment. This process can be formulated as follows:

$(g+p)(2 E)^{C t}=A$, where $g$ is the genomic DNA copy number, $p$ is the plasmid copy number, $C t$ is the PCR cycle number, $E$ is the PCR amplification coefficient, and $A$ is the number of amplified molecules. The nonlinear least-squares method was used to obtain the parameters $g, E$ and $A$ (R language; http://www.r-project.org) (see additional file 6 ). The qPCR analysis yielded the genomic DNA copy number in $1 \mu \mathrm{l}$ of the protein sample from the wild-type plant (Figure 4A, Table 1). SDS-PAGE analysis followed by CBB staining was used to determine the number of RBC-L molecules in $1 \mu \mathrm{l}$ of the wild-type protein sample (Figure 4B, Figure 4C and Table 1); recombinant RBC-L purified from Escherichia coli was used as a control. These results indicated that the wild-type protein sample had $2.63 \times 10^{8} \pm 0.15 \times 10^{8}$ (mean \pm s.d., $n=4$ ) molecules of RBC-L per cell. In plants, Rubisco consists of eight large and eight small subunits [13]. Therefore, each cell had $3.29 \times 10^{7}$ Rubisco complexes. Furthermore, we measured the chlorophyll content [14] in the protein sample from 3-week-old wild-type rosette leaves (Table 1). The molecular weights of chlorophyll $a$ and chlorophyll $b$ are 893 and 907, respectively. We determined that $1 \mu \mathrm{l}$ of the wild-type protein sample had 

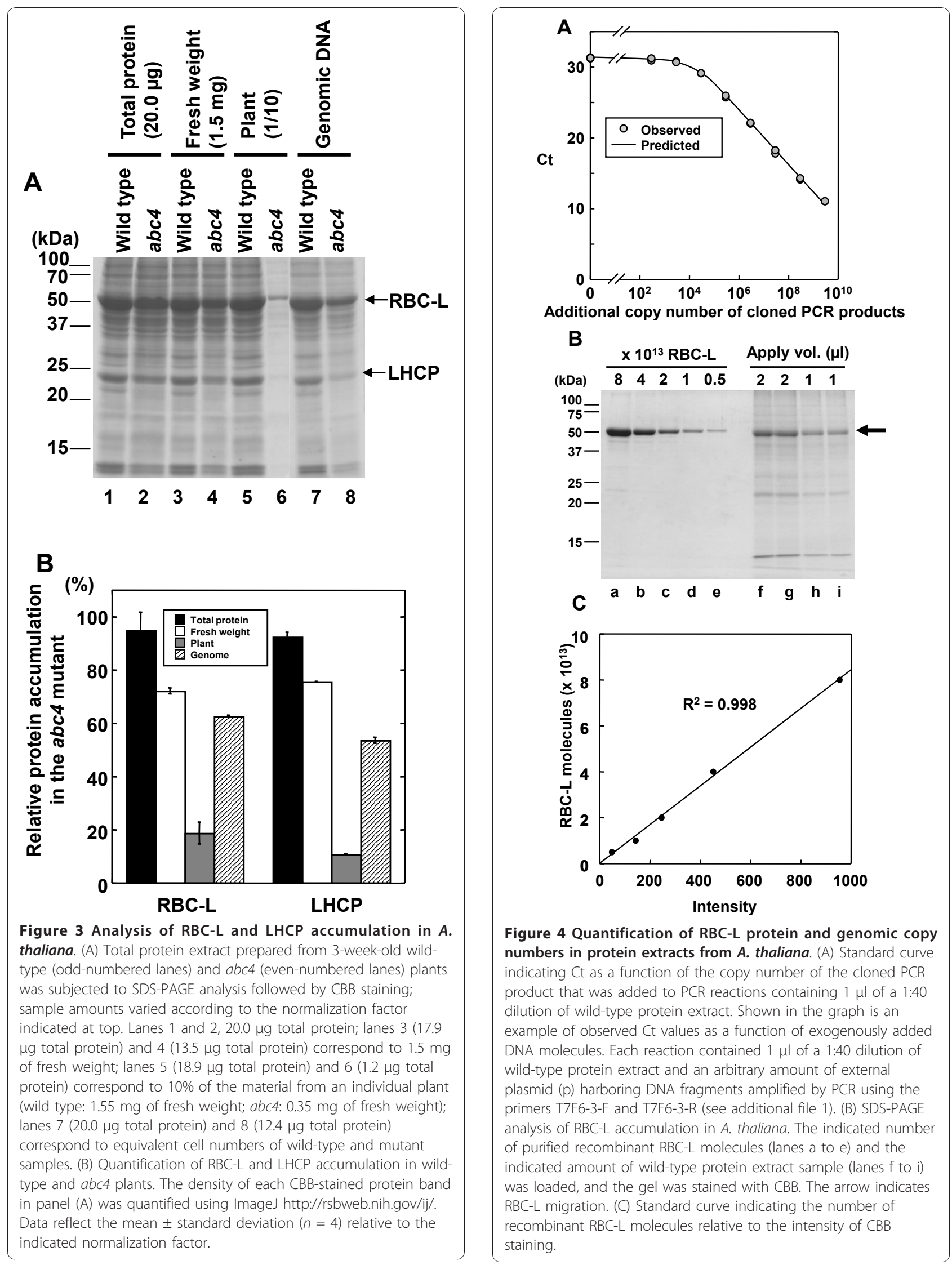
Table 1 Numbers of molecules in 3-week-old rosette leaves of wild-type Arabidopsis.

\begin{tabular}{|c|c|c|c|c|}
\hline Sample numbers & $\# 1$ & $\# 2$ & $\# 3$ & $\# 4$ \\
\hline Genomic copy number/ $\mu$ l & $2.47 \times 10^{5}$ & $2.40 \times 10^{5}$ & $4.12 \times 10^{5}$ & $4.39 \times 10^{5}$ \\
\hline s.e.m & $0.16 \times 10^{5}$ & $0.22 \times 10^{5}$ & $0.47 \times 10^{5}$ & $0.33 \times 10^{5}$ \\
\hline n & 11 & 10 & 20 & 20 \\
\hline RBC-L number/ $\mu l$ & $1.48 \times 10^{13}$ & $1.57 \times 10^{13}$ & $2.40 \times 10^{13}$ & $2.53 \times 10^{13}$ \\
\hline s.d. & $0.05 \times 10^{13}$ & $0.18 \times 10^{13}$ & $0.36 \times 10^{13}$ & $0.18 \times 10^{13}$ \\
\hline$n$ & 4 & 4 & 4 & 4 \\
\hline${ }^{1}$ RBC-L number/genome & $5.99 \times 10^{7}$ & $6.54 \times 10^{7}$ & $5.83 \times 10^{7}$ & $5.76 \times 10^{7}$ \\
\hline${ }^{2}$ RBC-L number/cell & $2.60 \times 10^{8}$ & $2.84 \times 10^{8}$ & $2.54 \times 10^{8}$ & $2.51 \times 10^{8}$ \\
\hline${ }^{3}$ RBC-L number/cell & $2.63 \times 10^{8}$ & & & \\
\hline s.d. & $0.15 \times 10^{8}$ & & & \\
\hline${ }^{4} \mathrm{CV}$ & $5.70 \times 10^{-2}$ & & & \\
\hline 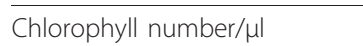 & $9.88 \times 10^{13}$ & $10.01 \times 10^{13}$ & $17.72 \times 10^{13}$ & $19.78 \times 10^{13}$ \\
\hline s.d. & $0.81 \times 10^{13}$ & $0.20 \times 10^{13}$ & $0.15 \times 10^{13}$ & $0.04 \times 10^{13}$ \\
\hline$n$ & 2 & 2 & 2 & 2 \\
\hline${ }^{5}$ Chlorophyll number/genome & $4.00 \times 10^{8}$ & $4.17 \times 10^{8}$ & $4.30 \times 10^{8}$ & $4.51 \times 10^{8}$ \\
\hline${ }^{6}$ Chlorophyll number/cell & $1.74 \times 10^{9}$ & $1.81 \times 10^{9}$ & $1.84 \times 10^{9}$ & $1.96 \times 10^{9}$ \\
\hline${ }^{3}$ Chlorophyll number/cell & $1.85 \times 10^{9}$ & & & \\
\hline s.d. & $0.09 \times 10^{9}$ & & & \\
\hline${ }^{4} \mathrm{CV}$ & $4.86 \times 10^{-2}$ & & & \\
\hline
\end{tabular}

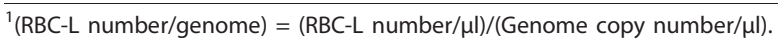

${ }^{2}($ RBC-L number/cell) $=($ RBC-L number/genome $) \times($ Genome copy number/cell).

$=($ RBC-L number/genome) $\times 4.35$.

${ }^{3}$ Mean of sample number 1 to 4 .

${ }^{4}$ Coefficient of variation (s.d./mean)

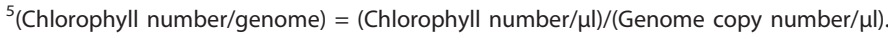

${ }^{6}($ Chlorophyll number/cell $)=($ Chlorophyll number/genome $) \times($ Genome copy number/cell $)$.

$=($ Chlorophyll number $/$ genome $) \times 4.35$.

between $9.88 \times 10^{13}$ and $1.98 \times 10^{14}$ chlorophyll molecules, indicating that each cell had $1.85 \times 10^{9} \pm 0.09 \times$ $10^{9}$ (mean \pm s.d., $n=4$ ) molecules of chlorophyll. The fact that the coefficient of variation for the number of RCB-L and chlorophyll molecules per genome was relatively low $\left(5.70 \times 10^{-2}\right.$ and $4.86 \times 10^{-2}$, respectively; Table 1) suggested that quantification based on the genomic DNA copy number was reproducible.

We quantified the amount of RBC-L according to the intensity of CBB staining following SDS/PAGE analysis. However, it is also possible to use immunoblotting or enzyme-linked immunosorbent assay to quantify the number of molecules of a particular protein per genomic DNA copy number.

\section{Conclusions}

In this study, we demonstrate that establishing an appropriate normalization factor is a challenging yet vital aspect of comparing protein, transcript, or metabolite levels among samples. Our results establish a facile and accurate method for quantifying these molecules based on genomic DNA copy number and ploidy. Although we performed cytometric analysis to measure the ploidy, a simplified method without the cytometric analysis may be applicable when assessing the effect(s) of a short-term treatment (e.g., induction of stress over several hours). Furthermore, our method can provide information on the number of transcripts, proteins, and metabolites per cell, and it should be applicable for stoichiometry and mathematical modeling of cellular systems.

\section{Methods}

\section{Plant materials}

Arabidopsis thaliana, Wassilewskija (wild type) and abc4 [9] (Wassilewskija background) were grown at $23^{\circ} \mathrm{C}$ under continuous light $\left(32.0 \mu \mathrm{mol} \cdot \mathrm{m}^{-2} \mathrm{~s}^{-1}\right)$ on plates containing $1 / 2$ MS medium and $1.5 \%$ sucrose.

\section{Ploidy measurement}

Flow cytometry was performed by a Ploidy Analyzer (Partec, Münster, Germany) [15]. At least 5,000 nuclei isolated from rosette leaves of each 3-week-old Arabidopsis plant were used for each ploidy measurement. Three biological and two technical replicates were used for each sample analyzed.

\section{Preparation of total nucleic acid from $A$. thaliana}

Rosette leaves $(100 \mathrm{mg})$ from each 3-week-old Arabidopsis plant were ground with a mortar and pestle in liquid 
nitrogen and homogenized in 5 volumes $(\mathrm{v} / \mathrm{w})$ of extraction buffer (100 mM MOPS-KOH, pH 7.0, 10 mM EDTA, $0.3 \mathrm{M} \mathrm{NaCl}, 1.0 \%$ SDS). Extraction of total nucleic acids (genomic DNA and total RNA) was performed by the addition of 5 volumes of phenol saturated with $1 \mathrm{M}$ MOPS$\mathrm{KOH}, \mathrm{pH} \mathrm{7.0,} \mathrm{and} 5$ volumes of chloroform:isoamyl alcohol $(24: 1, \mathrm{v} / \mathrm{v})$ followed by vigorous agitation and centrifugation for $10 \mathrm{~min}$ at $20,000 \times g$. The aqueous phase was collected and extracted two times with an equal volume of phenol:chloroform:isoamyl alcohol $(25: 24: 1, \mathrm{v} / \mathrm{v} / \mathrm{v})$. The nucleic acids were precipitated with ethanol and suspended in $50 \mu \mathrm{l}$ nuclease-free water (see additional file 7).

\section{Preparation of cDNA and genomic DNA}

To prepare cDNAs, $4 \mu \mathrm{l}$ total nucleic acid $\left(\mathrm{A}_{260}=20.0\right)$ was digested with Turbo DNA-free DNase I (Ambion, Austin, TX, USA) according to the manufacturer's instructions. Absence of genomic DNA in DNase I treated samples was verified by PCR using primers T7F6-F2 and T7F6-R-2 or MDC16-F-2 and MDC16-R-2 (see additional file 1). cDNA synthesis was performed using the PrimeScript RT reagent kit in the presence of oligo $\mathrm{dT}$ and random 6-mer primers according to the manufacturer's instructions (Takara Bio. Inc., Ohtsu, Japan). To prepare genomic DNA, $4 \mu \mathrm{l}$ total nucleic acid $\left(\mathrm{A}_{260}\right.$ $=20.0$ ) was digested with RNase (Wako Pure Chemical Industries, Osaka, Japan) (see additional files 7 and 8).

\section{Protein sample preparation}

Rosette leaves (100 mg) from each 3-week-old plant were ground with a mortar and pestle in liquid nitrogen and transferred to a new 2-ml tube and homogenized in 5 volumes $(\mathrm{v} / \mathrm{w})$ of extraction buffer containing $15 \mathrm{mM}$ Tris- $\mathrm{HCl}, \mathrm{pH}$ 8.0, $50 \mathrm{mM} \mathrm{NaCl}, 10 \mathrm{mM}$ EDTA, 1.0\% SDS and $1.0 \%$ protease inhibitor cocktail (SigmaAldrich, Tokyo, Japan). The samples were incubated on ice for $10 \mathrm{~min}$ with vigorous vortexing every minute. The samples were centrifuged for $5 \mathrm{~min}$ at 10,000 $\times g$, and the supernatants were used for further experiments. The total protein concentration of each supernatant was determined using a Coomassie Protein Assay kit (Pierce Biotechnology, Rockford, IL, USA). Chlorophyll concentrations were calculated according to Arnon [14].

\section{qPCR analysis}

All qPCR reactions were analyzed with an ABI PRISM 7300 sequence detection system (Applied Biosystems, Foster City, CA, USA), and data were analyzed using SDS 2.2.1 software (Applied Biosystems). The KAPA SYBR FAST qPCR kit (Kapa Biosystems, Boston, MA, USA) was used for qPCR amplification of purified genomic DNA or cDNA according to the manufacturer's instructions; the $20-\mu \mathrm{l}$ reactions contained $1 \mu \mathrm{l}$ of genomic DNA or cDNA template and $8 \mathrm{pmol}$ of each set of gene-specific primers (see additional files 1 ). PCR reaction conditions were as follows: $95^{\circ} \mathrm{C}$ for $3 \mathrm{~min}$, followed by 40 cycles of $95^{\circ} \mathrm{C}$ for $15 \mathrm{~s}$ and $60^{\circ} \mathrm{C}$ for $40 \mathrm{~s}$. Transcript levels were quantified by the $\Delta \Delta$ Ct method [11]. The KAPA2G Robust HotStart kit (Kapa Biosystems) was used for qPCR amplification of genomic DNA in protein extract supernatants; the $20-\mu$ l reactions contained $4 \mu \mathrm{l}$ of undiluted KAPA2G Buffer B, $4 \mu \mathrm{l}$ of undiluted Enhancer 1, $1.6 \mu \mathrm{l}$ of $2.5 \mathrm{mM}$ dNTP, 8 pmol of each gene-specific primer (T7F6-3-F and T7F6$3-\mathrm{R}$, see additional file 1 ), $1 \mu \mathrm{l}$ of $0.1 \%$ SYBR Green I (Takara), 0.4 $\mu$ l Rox High (from the KAPA SYBR FAST qPCR kit), 2 U of KAPA2G Robust HotStart DNA polymerase, and $1 \mu \mathrm{l}$ of protein extract. For quantification of genomic DNA copy number in the mutant plants relative to wild-type plants, $1 \mu \mathrm{l}$ of protein extract of wild-type plants (diluted 1:10, 1:20, 1:40, 1:80 or 1:160) was added to the PCR reactions, and a standard curve was generated by qPCR (see additional file 5). One microliter of a 1:40 dilution of protein extract of the mutant plants was added to the PCR reactions, and qPCR was performed. For absolute quantification of genomic DNA copy number in protein extracts of wild-type plants, $1 \mu \mathrm{l}$ of a 1:40 dilution of wildtype protein extract and $1 \mu \mathrm{l}$ of cloned PCR products (between $2.88 \times 10^{2}$ and $2.88 \times 10^{9}$ molecules, or 0 molecules) was added to the PCR reactions; the 20- $\mu$ l reactions contained $4 \mu \mathrm{l}$ of undiluted KAPA2G Buffer B, $4 \mu \mathrm{l}$ of undiluted Enhancer 1, 4.4 $\mu \mathrm{l}$ of $25 \mathrm{mM} \mathrm{MgCl}_{2}, 1.6 \mu \mathrm{l}$ of $2.5 \mathrm{mM}$ dNTP, $8 \mathrm{pmol}$ of each gene-specific primer (T7F6-3-F and T7F6-3-R, see additional file 1), 10 pmol of TaqMan Probe (see additional file 1), $0.4 \mu \mathrm{l}$ Rox High (from the KAPA SYBR FAST qPCR kit), 2 U of KAPA2G Robust HotStart DNA polymerase, and $1 \mu \mathrm{l}$ of protein extract, and PCR was performed. This dilution series was prepared with EASY Dilution (for real-time PCR) (Takara). PCR reaction conditions were as follows: $95^{\circ} \mathrm{C}$ for $3 \mathrm{~min}$, followed by 40 cycles of $95^{\circ} \mathrm{C}$ for $15 \mathrm{~s}$ and $72^{\circ} \mathrm{C}$ for $40 \mathrm{~s}$.

\section{Preparation of cloned PCR products}

PCR was performed using primers T7F6-3-F and T7F6-3-R (see additional file 1) with genomic DNA of wild-type Arabidopsis as template. The amplified DNA fragment was ligated into the TA cloning vector, pMD20 (Takara), and the sequence was confirmed. The plasmids containing the PCR products were digested with $X h o I$ and purified. The mass of a nucleotide pair in DNA is $660 \mathrm{Da}$, and the plasmid containing the PCR product was $2,872 \mathrm{bp}$. The concentration of the linearized plasmid was determined, and the number of plasmid molecules was calculated.

\section{Expression and purification of RBC-L}

The full-length open reading frame of RBC-L was amplified by PCR using primers containing an NdeI site (RBCL-Nde, 5'-CCCCATATGTCACCACAAACAGAGACTAAAG-3'; Nde I site underlined) and a XhoI site 
(RBCL2-Xho, 5'-CCCCTCGAGCTCTTGGCCATCTAATTTATCGATG-3'; XhoI site underlined). The amplified DNA fragment was digested with NdeI and $X$ XoI and ligated into the expression vector pET-24a $(+)$ (Novagen, San Diego, CA, USA). RBC-L was expressed and purified as described [16]. The molecular weight of RBC-L was 54,019. The concentration of the purified $\mathrm{RBC}-\mathrm{L}$ was determined, and the number of RBC-L molecules was calculated.

\section{Additional material}

Additional File 1: Primers used in this work. Table shows the primer names and the sequences.

Additional File 2: qPCR amplification and dissociation curves. (A)

Schematic representation of each PCR-amplified region in chromosomes I-V (see additional file 1). (B) Real-time GPCR amplification curves generated using equal volume of template and the primer sets indicated. The curves using the different primer sets were the same, suggesting that the amplification efficiency using three different primer sets and the extraction efficiency of the different regions of genomic DNA were essentially equivalent. (C) Dissociation curves for the PCR products generated using the indicated primer sets. The $y$ axis shows the logarithm of fluorescence. These curves reflect normalized data.

Additional File 3: Dissociation curves. Dissociation curves for the PCR products generated using the primer set of T7F6-F-2 and T7F6-R-2 (A), MDC16-F-2 and MDC16-R-2 (B), 18S-3-F and 18S-3-R (C), RBCL-2-F and RBCL2-R (D), or RBCS-3-F and RBCS-3-R (E) (see additional file 1). The $y$ axis shows the logarithm of fluorescence. These curves reflect normalized data.

Additional File 4: Transcript number per cell in 3-week-old rosette leaves of wild-type Arabidopsis. Table shows the transcript number of RBC-L, RBC-S, 18S, ACT2, PDF2, SAND, GAPDH, UBC, EF-1 $\alpha, P P R, Y L S 8$ and UBC9 genes per cell.

Additional File 5: Standard curve indicating the $\mathrm{Ct}$ relative the dilution of protein extract sample from a wild-type plant as template for qPCR. One microliter of wild-type protein extract (diluted $1: 10,1: 20,1: 40,1: 80$, or 1:160) was used as template for $\mathrm{qPCR}$

Additional File 6: Calculation of genomic DNA copy number in protein extracts. This file shows mathematical formula with actual command sequences on $\mathrm{R}$ language to calculate genomic DNA copy number.

Additional File 7: Scheme for preparing CDNA and genomic DNA Scheme shows the methods of extraction of the nucleic acid (genomic DNA and total RNA), DNase I digestion, reverse transcription and RNase digestion, and the extraction buffer composition.

Additional File 8: Agarose gel analysis of total nucleic acid genomic DNA, and total RNA preparations. Total nucleic acid from wild-type plants was treated without (lane 1) or with RNase (lane 2) or DNase (lane 3) and subjected to 1.2\% agarose gel electrophoresis followed by ethidium bromide staining.

\section{List of abbreviations}

LHCP: light-harvesting chlorophyll a/b protein; qPCR: quantitative PCR; qRT$P C R$ : quantitative reverse transcription $P C R$; $R B C$ or Rubisco: ribulose 1,5bisphosphate carboxylase/oxygenase; RBC-L: large subunit of Rubisco; RBC-S: small subunit of Rubisco; ACT2: actin 2; PDF2: transposable element gene; SAND: SAND family protein; GAPDH: glyceraldehyde 3-phosphate dehydrogenase; UBC: ubiquitin-conjugating enzyme; EF-1a: elongation factor 1-a; PPR: pentatricopeptide repeat-containing protein; YLS8: yellow-leafspecific protein 8; UBC9: ubiquitin-conjugation enzyme E2.

\section{Acknowledgements}

We thank T. Shibata (Hiroshima University) for critical reading of the manuscript. The Plant culture facility was provided by the Center for Gene Science, Hiroshima University. This work was supported by the Japan Society of the Promotion of Science [Grant-in-Aid for Scientific Research (c) (No. 20570032 to HS)].

\section{Author details}

${ }^{1}$ Department of Mathematical and Life Sciences, Graduate School of Science, Hiroshima University, 1-3-1, Kagamiyama, Higashi-Hiroshima, 739-8526, Japan. ${ }^{2}$ Graduate School of Information Sciences, Tohoku University, 6-3-09, Aramaki-Aza-Aoba, Aoba-ku, Sendai 980-8579, Japan. ${ }^{3}$ Plant Functional Genomics Research Group, Plant Science Center, RIKEN Yokohama Institute, 1-7-22 Suehiro-cho, Tsurumi-ku, Yokohama, 230-0045 Japan.

\section{Authors' contributions}

HS conceived and directed this study, performed all experimental work, and wrote the paper with input from co-authors. TO calculated the copy number of genomic DNA in protein samples. NT and MM performed the flow cytometry analysis. AS contributed to data analysis and provided advice. All authors read and approved the final manuscript.

\section{Competing interests}

The authors declare that they have no competing interests.

Received: 1 October 2010 Accepted: 29 December 2010

Published: 29 December 2010

\section{References}

1. Czechowski T, Stitt M, Altmann T, Udvardi MK, Scheible WR: Genome-wide identification and testing of superior reference genes for transcript normalization in Arabidopsis. Plant Physiol 2005, 139:5-17.

2. Guenin S, Mauriat M, Pelloux J, Van Wuytswinkel O, Bellini C, Gutierrez L: Normalization of qRT-PCR data: the necessity of adopting a systematic, experimental conditions-specific, validation of references. J Exp Bot 2009, 60:487-493.

3. Vandesompele J, De Preter K, Pattyn F, Poppe B, Van Roy N, De Paepe A, Speleman F: Accurate normalization of real-time quantitative RT-PCR data by geometric averaging of multiple internal control genes. Genome Biol 2002, 3:RESEARCH0034

4. Pfaffl MW, Tichopad A, Prgomet C, Neuvians TP: Determination of stable housekeeping genes, differentially regulated target genes and sample integrity: BestKeeper-Excel-based tool using pair-wise correlations. Biotechnol Lett 2004, 26:509-515.

5. Bilban M, Buehler LK, Head S, Desoye G, Quaranta V: Normalizing DNA microarray data. Curr Issues Mol Biol 2002, 4:57-64.

6. Salit M: Standards in gene expression microarray experiments. Methods Enzymol 2006, 411:63-78.

7. Hansen MC, Nielsen AK, Molin S, Hammer K, Kilstrup M: Changes in rRNA levels during stress invalidates results from mRNA blotting: fluorescence in situ rRNA hybridization permits renormalization for estimation of cellular mRNA levels. J Bacteriol 2001, 183:4747-4751.

8. Solanas M, Moral R, Escrich E: Unsuitability of using ribosomal RNA as loading control for Northern blot analyses related to the imbalance between messenger and ribosomal RNA content in rat mammary tumors. Anal Biochem 2001, 288:99-102.

9. Shimada H, Ohno R, Shibata M, Ikegami I, Onai K, Ohto MA, Takamiya K. Inactivation and deficiency of core proteins of photosystems I and II caused by genetical phylloquinone and plastoquinone deficiency but retained lamellar structure in a T-DNA mutant of Arabidopsis. Plant $J$ 2005, 41:627-637.

10. Inze D, De Veylder L: Cell cycle regulation in plant development. Annual Review of Genetics 2006, 40:77-105.

11. Livak KJ, Schmittgen TD: Analysis of relative gene expression data using real-time quantitative PCR and the 2(-Delta Delta C(T)) Method. Methods 2001, 25:402-408.

12. Liao TH: Reversible Inactivation of Pancreatic Deoxyribonuclease-a by Sodium Dodecyl-Sulfate - Removal of Cooh-Terminal Residues from Denatured Protein by Carboxypeptidase-A. Journal of Biological Chemistry 1975, 250:3831-3836. 
13. Spreitzer RJ, Salvucci ME: Rubisco: structure, regulatory interactions, and possibilities for a better enzyme. Annu Rev Plant Biol 2002, 53:449-475.

14. Arnon DI: Copper Enzymes in Isolated Chloroplasts. Polyphenoloxidase in Beta Vulgaris. Plant Physiol 1949, 24:1-15.

15. Takahashi N, Lammens T, Boudolf V, Maes S, Yoshizumi T, De Jaeger G, Witters E, Inze D, De Veylder L: The DNA replication checkpoint aids survival of plants deficient in the novel replisome factor ETG1. EMBO J 2008, 27:1840-1851.

16. Shimada H, Mochizuki M, Ogura K, Froehlich JE, Osteryoung KW, Shirano Y, Shibata D, Masuda S, Mori K, Takamiya K: Arabidopsis cotyledon-specific chloroplast biogenesis factor $\mathrm{CYO} 1$ is a protein disulfide isomerase. Plant Cell 2007, 19:3157-3169.

doi:10.1186/1746-4811-6-29

Cite this article as: Shimada et al.: Normalization using ploidy and genomic DNA copy number allows absolute quantification of transcripts, proteins and metabolites in cells. Plant Methods 2010 6:29.

\section{Submit your next manuscript to BioMed Central} and take full advantage of:

- Convenient online submission

- Thorough peer review

- No space constraints or color figure charges

- Immediate publication on acceptance

- Inclusion in PubMed, CAS, Scopus and Google Scholar

- Research which is freely available for redistribution

Submit your manuscript at www.biomedcentral.com/submit
Ciomed Central 